\title{
REC8 inhibits EMT by downregulating EGR1 in gastric cancer cells
}

\author{
JUNHONG ZHAO $^{1}$, LANLAN GENG $^{1}$, GAOYANG DUAN $^{1}$, WANFU XU ${ }^{1}$, \\ YANG CHENG $^{1}$, ZHILIANG HUANG ${ }^{2}$, ZHENWEN ZHOU $^{1}$ and SITANG GONG ${ }^{1}$ \\ ${ }^{1}$ Department of Gastroenterology, Guangzhou Women and Children's Medical Center, Guangzhou Medical University, \\ Guangzhou, Guangdong 510120; ${ }^{2}$ Cancer Center of Guangzhou Medical University, \\ Guangzhou, Guangdong 510089, P.R. China
}

Received June 20, 2017; Accepted December 7, 2017

DOI: $10.3892 / o r .2018 .6244$

\begin{abstract}
REC8 is a component of the meiotic cohesion complex that plays a critical role in chromosome dynamics during meiosis. However, the functional role of REC8 in gastric cancer has not been elucidated. In the present study, REC8 suppressed the growth and metastasis of gastric cancer cells in vitro. Whole Human Genome Oligo Microarray results revealed that a wide range of genes with broad function were targeted by REC8. Among them early growth response-1 (EGR1), a transcription factor and an epithelial-mesenchymal transition (EMT)-associated protein in the AGR-RAGE pathway was significantly downregulated when REC8 was overexpressed in gastric cancer cells. We hypothesized that REC8 inhibits EMT by downregulating EGR1 in gastric cancer cells. Consistent with our prediction, REC8 overexpression decreased EMT in gastric cancer cells, whereas the REC8 ablation reversed these effects. In addition, the phenotypes of EGR1 overexpressed cells were similar to the phenotypes of REC8 ablated cells. Furthermore, we determined that REC8 interacted with EGR1, and inhibited EMT in gastric cancer cells. We thus propose further studies of the pathways associated with REC8 and EGR1 to potentially find novel targets in the treatment for gastric cancer.
\end{abstract}

\section{Introduction}

Gastric cancer is a malignant disease which has a high mortality rate and is the second leading cause of cancer-

Correspondence to: Dr Sitang Gong, Department of Gastroenterology, Guangzhou Women and Children's Medical Center, Guangzhou Medical University, 318 Renminzhong Road, Guangzhou, Guangdong 510120, P.R. China

E-mail: sitangg@126.com

Abbreviations: REC8, meiotic recombination protein REC8 homolog; EGR1, early growth response-1; EMT, epithelialmesenchymal transition

Key words: REC8, gastric cancer, EMT, EGR1 related deaths worldwide (1). Although recent advances in surgery, chemotherapy, and radiation therapy have improved the outcome of gastric cancer, patients who undergo curative surgical resection often succumb to recurrent disease due to tumor invasion and metastasis. Hence, to treat gastric cancer, it is necessary to elucidate the molecular mechanisms involved in the development and invasiveness of gastric cancer.

Epithelial-mesenchymal transition (EMT) is a physiological and pathological process where epithelial cells lose their polarity and cell-cell adhesion signature, and acquire the characteristics of mesenchymal cells (2). EMT plays a critical role in many aspects of cancer behavior, including phenotypic conversion that is implicated in the initiation of metastasis in gastric cancer progression (3). During EMT, the expression of some epithelial cell markers, such as E-cadherin decreases, while the expression of mesenchymal cell markers, such as vimentin increases. In addition, the EMT-related transcription factor, SOX10 (4), is upregulated, during this process. The $\mathrm{Wnt} / \beta$-catenin signaling pathway is also associated with EMT; in fact, aberrant $\beta$-catenin subcellular localization expression is considered a surrogate marker of aberrant Wnt signaling pathway activation.

REC8 is a key meiosis-specific component of the cohesion complex that binds sister chromatids in preparation for the two divisions of meiosis. REC8 has also been implicated in DNA damage repair and maintenance of chromosome stability (5). Previous studies revealed that REC8 was hypermethylated in melanomas and malignant gastrointestinal stromal tumors. Moreover, REC8 was associated with poor tumor prognosis in patients (6-8). The novel role of REC8 as a tumor-suppressor gene was recently identified through the robust epigenetic inactivation of REC8 by PI3K. REC8 was also revealed to play an important role in many human cancers (9). These results indicated that REC8 has the potential to suppress tumors in gastric cancer.

We thus hypothesized that REC8 and EMT are involved in the progression of gastric cancer. We determined that REC8 inhibited the proliferation, migration and invasion of gastric cancer cells in vitro. Furthermore, we used genome-wide expression microarray to analyze the association of REC8 expression with differential gene expression profiles and determined that the expression of early growth response-1 (EGR1), 
a transcription factor mediated by the AGE-RAGE signaling pathway (10) was regulated by REC8. However, the role of EGR1 on EMT remains unclear due to opposite functions that have been reported in studies $(11,12)$.

In the present study, we determined the function of REC8 by assessing gain- and loss-of-function of REC8 and its effect on EMT in gastric cancer cells. Moreover, we revealed that REC8 expression regulated the expression of EGR1 and mediated EMT. Finally, we assessed the manner in which REC8 regulated the expression of EGR1, specifically through its interaction. Therefore, we demonstrated the effects of REC8 on gastric cancer cell proliferation and migration, and revealed that REC8 inhibited cell EMT by downregulating EGR1 in gastric cancer via REC8 and ERG1 interactions.

\section{Materials and methods}

Cell culture. The BGC823 and SGC-7901 cell lines were obtained from the Cell Bank of the Chinese Academy of Sciences (Chinese Academy of Sciences, Shanghai, China). Cells were routinely grown in RPMI-1640 medium supplemented with $10 \%(\mathrm{v} / \mathrm{v})$ fetal bovine serum (FBS) at $37^{\circ} \mathrm{C}$ in a humidified atmosphere of $5 \% \mathrm{CO}_{2}$.

REC8 shRNA, REC8 and EGRI overexpression constructs and infection. REC8 shRNA was purchased from Origene Technologies, Inc. (cat. no. TG309883; Rockville, MD, USA). For the overexpression of REC8 and EGR1, full-length homo sapiens REC8 and EGR1 sequences (based on NCBI) were cloned into the pcDNA3.1 vector. The shRNA plasmid pcDNA3.1-REC8 or pcDNA3.1-EGR1 were transiently transfected into cells using Lipofectamine 2000 reagent (cat. no. 11668019; Invitrogen/Thermo Fisher Scientific, Inc., Waltham, MA, USA) according to the manufacturer's instructions.

Cell proliferation analysis. Cells $\left(1 \times 10^{4}\right)$ transfected with vector or pcDNA3.1-REC8 were seeded into 96-well culture plates and cultured for $72 \mathrm{~h}$. At 0, 24, 48 and $72 \mathrm{~h}, \mathrm{CCK}-8$ reagent was added to each well, according to the manufacturer's instructions (cat. no. 96992; Sigma-Aldrich, St. Louis, MO, USA). The optical density values (OD values) were assessed at $450 \mathrm{~nm}$ using a plate reader (Thermo Scientific ${ }^{\mathrm{TM}}$ Multiskan $^{\mathrm{TM}}$ GO; Thermo Fisher Scientific).

Wound healing assay. Cells $\left(1 \times 10^{5}\right)$ were seeded and cultured in a 24-well plate. After the cells were confluent, the cell layer was carefully wounded using sterile tips $(200 \mu \mathrm{l})$. The falling cells were washed off with phosphate-buffered saline (PBS). After incubation for 0 and $48 \mathrm{~h}$ in serum-free medium, the images of the cells were captured by light microscope at a low magnification (4x, objective) and wound healing was assessed.

Transwell invasion assays. The precoated Transwell filters ( $8-\mu \mathrm{m}$ pore size) were set in the 24 -well plate. After being serum-starved for $12 \mathrm{~h}$, the BGC823 cells $\left(1 \times 10^{5}\right)$ were loaded into the upper chamber. Conditioned medium containing $10 \%$ FBS was added to each of the wells, and the cells were allowed to invade by penetrating through the membrane during a $24-\mathrm{h}$ incubation period at $37^{\circ} \mathrm{C}$. The cells on the lower surface of the Transwell filters were stained with $5 \%$ crystal violet. The results were calculated from five random fields taken by light microscope.

Microarrays and gene expression analysis. Total RNA was extracted using TRIzol reagent, according to the manufacturer's instructions. Whole Human Genome Oligo Microarray (Agilent Technologies, Inc., Santa Clara, CA, USA) was performed by KangChen Bio-tech (Shanghai, China) to observe the difference in transcriptional profiles between the pcDNA3.1-vector and -REC8 cells. The microarray datasets revealed the differential expression of genes and these were quantile normalized and analyzed by the GeneSpring GX software package (Version 11.0; Agilent Technologies). The Gene Ontology (GO) biological process and Kyoto Encyclopedia of Genes and Genomes (KEGG) pathway enrichment analysis were performed. The Database for Annotation, Visualization and Integrated Discovery (DAVID) 6.7 was used and the results were ranked by P-values.

cDNA preparation and real-time PCR. Total RNA was extracted using TRIzol reagent, according to the manufacturer's instructions. For quantitative reverse-transcription polymerase chain reaction (qRT-PCR), total RNA was reverse-transcribed to cDNA by using Bestar qPCR RT kit (cat. no. DBI-2220; DBI Bioscience, Ludwigshafen, Germany). Next, cDNA was quantified by real-time PCR on an Mx3000P real-time PCR system (Agilent Technologies) with DBI Bestar ${ }^{\circledR}$ SYBR Green qPCR Master Mix (cat. no. DBI-2043; DBI Bioscience). Results were normalized to the expression of $\beta$-actin. The $2^{-\Delta \Delta \mathrm{Ct}}$ method was used for calculating expression levels. The PCR primers are shown in Table I.

Protein extraction and western blot analysis. Cells were collected and lysed on ice in RIPA buffer containing a protease inhibitor cocktail. Protein concentration was assessed and $100 \mu \mathrm{g}$ of protein from each sample was loaded and separated by $10 \%$ polyacrylamide gel electrophoresis and transferred onto PVDF membranes. After incubation with the specific primary antibodies: REC8 antibody (1:1,000; rabbit monoclonal, cat. no. ab192241; Abcam, San Francisco, CA, USA), EGR1 antibody (1:1,000; rabbit polyclonal, cat. no. ab6054; Abcam), TGF $\beta 1$ antibody (1:1,000; rabbit polyclonal, cat. no. ab92486; Abcam), SOX10 antibody (1:1,000; rabbit polyclonal, cat. no. ab108408; Abcam), vimentin antibody $(1: 1,000$; rabbit monoclonal, cat. no. ab92547; Abcam), E-cadherin antibody (1:1,000; rabbit polyclonal, cat. no. 3195; Cell Signaling Technology, Danvers, MA, USA), GAPDH antibody (1:4,000; rabbit polyclonal, cat. no. 2118; Cell Signaling Technology), ATG12 antibody (1:1,000; mouse monoclonal, cat. no. sc-271688; Santa Cruz Biotechnology, Santa Cruz, CA, USA) at $4^{\circ} \mathrm{C}$ overnight, the membranes were incubated with HRP-conjugated secondary antibodies (1:10,000; cat no. A4416 and A6154; SigmaAldrich). ECL luminescent reagent (cat. no. 32109; Invitrogen/ Thermo Fisher Scientific) was added and the signals were recorded on X-ray film in a darkroom. GAPDH was used as the loading control.

Immunofluorescence assay. Cells were fixed with $4 \%$ paraformaldehyde and incubated with anti- $\beta$-catenin antibody (1:100; rabbit monoclonal $\beta$-catenin antibody, cat. no. 8480; 
Table I. Primers used in qRT-PCR.

ID

Sequences $\left(5^{\prime}-3^{\prime}\right)$

Product length (bp)

$\begin{array}{ll}\text { B-actin Forward } & \text { ATCGTGCGTGACATTAAGGAGAAG } \\ \text { B-actin Reverse } & \text { AGGAAGGAAGGCTGGAAGAGTG } \\ \text { REC8 Forward } & \text { CATCCCACCAGAAGAACGG } \\ \text { REC8 Reverse } & \text { GCACCAAAGGCATCTCCAT } \\ \text { EGR1 Forward } & \text { TTCGACCTGCTCATCTTCGG } \\ \text { EGR1 Reverse } & \text { CGATGCGTGAGTCCATGTGT } \\ \text { TGF } \beta 1 \text { Forward } & \text { GCAACAATTCCTGGCGATAC } \\ \text { TGF } 1 \text { Reverse } & \text { AAGGCGAAAGCCCTCAAT } \\ \text { ATG12 Forward } & \text { TAGAGCGAACACGAACCATCC } \\ \text { ATG12 Reverse } & \text { CACTGCCAAAACACTCATAGAGA } \\ \text { SOX10 Forward } & \text { CCTCACAGATCGCCTACACC } \\ \text { SOX10 Reverse } & \text { CATATAGGAGAAGGCCGAGTAGA } \\ \text { E-cadherin Forward } & \text { CCCTGTTGGTGTCTTTATTATTG } \\ \text { E-cadherin Reverse } & \text { ATTCGGGCTTGTTGTCATTC } \\ \text { Vimentin Forward } & \text { GACGCCATCAACACCGAGTT } \\ \text { Vimentin Reverse } & \text { CTTTGTCGTTGGTTAGCTGGT }\end{array}$

Cell Signaling Technology) overnight at $4^{\circ} \mathrm{C}$. After blocking and washing, the coverslips were incubated with anti-rabbit IgG $(\mathrm{H}+\mathrm{L}) \mathrm{F}\left(\mathrm{ab}^{\prime}\right)_{2}$ Fragment (Alexa Fluro 488 conjugate) (1:500; cat. no. 4412; Cell Signaling Technology). The nuclei were stained with DAPI (4',6-diamidino-2-phenylindole) for $5 \mathrm{~min}$. After 3 washes, the slides were immediately examined with a confocal microscope (Zeiss 710, 63x oil; Carl Zeis, Heidenheim, Germany) and the images were captured.

Immunoprecipitation assays. Cell lysates were incubated with protein-A/G linked agarose $(1 \mathrm{mg}$ of protein $/ 40 \mu \mathrm{l}$ of beads) for $1 \mathrm{~h}$ at $4^{\circ} \mathrm{C}$ to exclude any non-specific binding. The supernatant collected after centrifugation was incubated with $1 \mu \mathrm{g}$ of the specific antibody REC8 (1:1,000; rabbit monoclonal, cat. no. ab192241; Abcam) overnight at $4^{\circ} \mathrm{C}$. The solution was then incubated with protein-A/G agarose beads for specific binding. IgG was used as the negative control. Beads were washed with RIPA buffer after incubation and dissolved in an SDS-PAGE loading buffer. The subsequent steps were performed as in the western blot analysis and have been previously described.

Statistical analysis. All data were obtained from at least three independent experiments. The significance of the differences between groups was determined using a two-tailed Student's- $t$ test or analysis of variance (ANOVA) followed by Tukey's test. $\mathrm{P}<0.05$ was considered to indicate a statistically significant result.

\section{Results}

Overexpression of REC8 inhibits the proliferation, migration and invasion of gastric cancer cells. To assess the effect of REC8 on gastric cancer cell proliferation, migration and invasion, we overexpressed REC 8 by transient transfection in BGC823 cells. Cells overexpressing REC8 protein were successfully obtained, as observed by the RT-PCR (Fig. 1A) and western blotting data (Fig. 1B).

We assessed tumor cell proliferation in response to REC8 overexpression, using the CCK-8 assay in BCG823 cell lines. As shown in Fig. 1C, the BGC823 cells overexpressing REC8 exhibited significantly reduced proliferation compared with vector controls at 48 and $72 \mathrm{~h}(\mathrm{P}=0.0401$ and $\mathrm{P}=0.0295)$. We further examined the effect of REC8 overexpression on the motility of BGC823 cells using the scratch wound healing assay. As shown in Fig. 1D and F, the migration of BGC823 and SGC-7901 cells overexpressing REC8 was significantly reduced $(\mathrm{P}<0.001)$. In addition, we examined the invasion of BGC823 and SGC-7901 cells overexpressing REC8 using a chamber invasion assay, and found that the invasion rates of these cells were also decreased $(P=0.0002$; Fig. $1 E$ and $G)$.

Overexpression of REC8 induces the AGE-RAGE pathway. We used genomic microarray to analyze the transcriptome profiles induced by REC 8 expression in BGC 823 cells. A total of 1,657 genes were differentially expressed by at least 2-fold between BGC823-REC8 and -pcDNA3.1 cells. We analyzed the biological processes of the downregulated genes using GO and the KEGG pathway databases with bioinformatic methods (Fig. 2A and B). Among the 9 significantly enriched KEGG terms and/or pathways (Table II), the AGE-RAGE signaling pathway is associated with EMT, while the FoxO signaling pathway is associated with apoptosis. The expression of EGR1 and TGF $\beta 1$ in the AGE-RAGE pathway and the expression of ATG12 and TGF $\beta 1$ in the FoxO pathway were significantly downregulated by overexpression of REC8. The qRT-PCR and western blot assays further validated the results of the microarray (Fig. $2 \mathrm{C}$ and $\mathrm{D} ; \mathrm{P}=0.0025, \mathrm{P}=0.0157$ and $\mathrm{P}=0.0023$, respectively). Since the expression of EGR1 was downregulated significantly, we hypothesized that the AGE-RAGE signaling pathway should be further investigated in future research. 

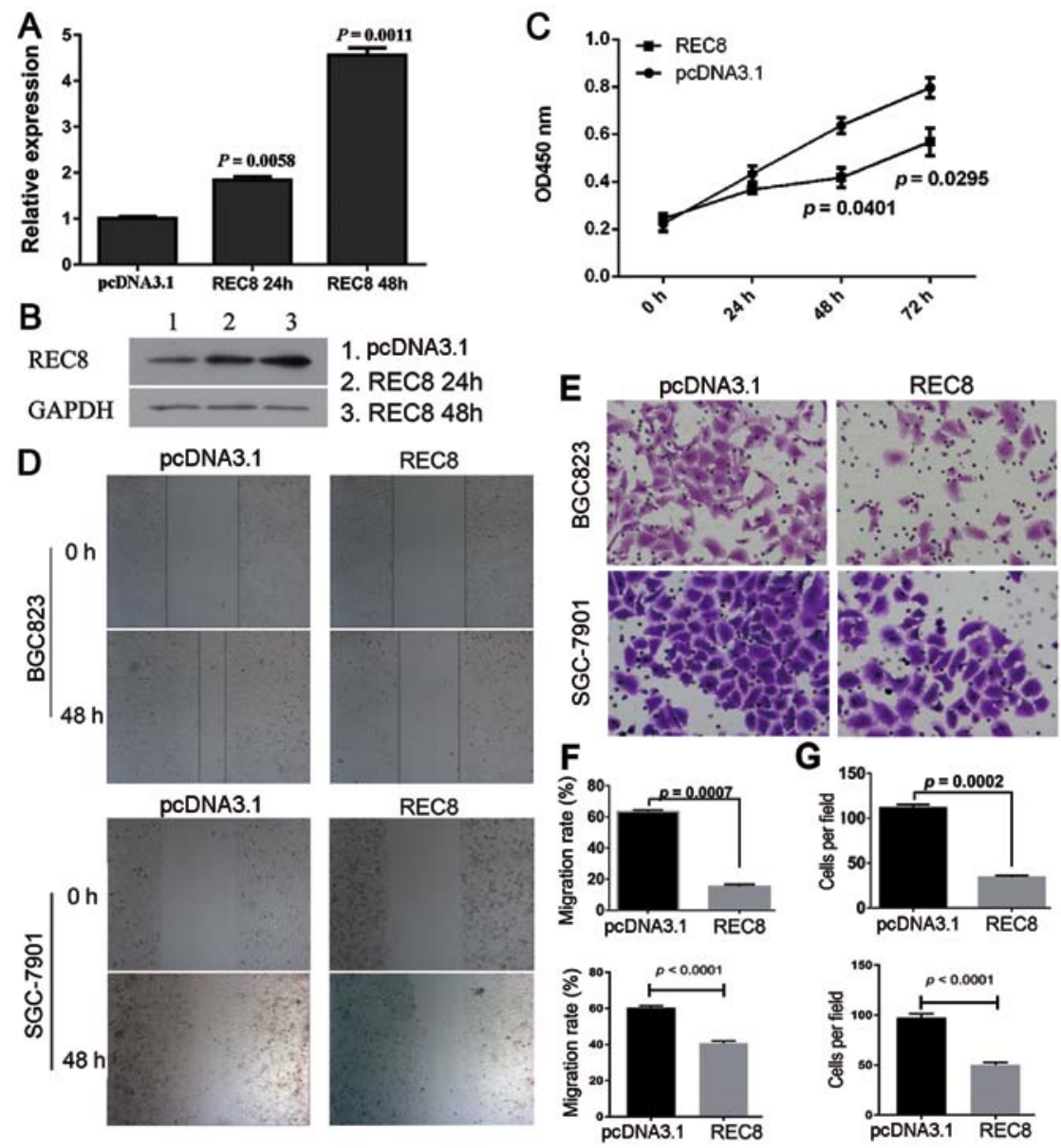

Figure 1. Overexpression of REC8 inhibits the proliferation, migration and invasion of gastric cancer cells. Gastric cancer cells BGC823 were transiently transfected with REC8 overexpression plasmid. (A and B) The overexpression of REC8 in BGC823 cells was assessed using qRT-PCR and western blotting. (C) The CCK-8 assay was used to determine cell proliferation of BGC823 cells after transfection with REC8 every 24 h for 3 days. Overexpression of REC8 inhibited the proliferation of gastric cancer cells. ( $\mathrm{D}$ and F) Cell migration was determined using the wound healing migration assay (magnification, $\mathrm{x} 40$ ) and the width of wound was assessed using a microscope in BGC823 and SGC-7901 cell lines. (E and G) Cell invasion was determined using Transwell invasion assay and the number of cells was counted. All the results were expressed as the mean \pm SD of three independent experiments with triplicate wells.

Table II. KEGG pathway enrichment analysis of the expression of downregulated genes between BGC823-REC8 and -pcDNA3.1 cells.

\begin{tabular}{llll}
\hline Pathway ID & \multicolumn{1}{c}{ Definition } & P-value (Fisher) & \multicolumn{1}{c}{ Genes } \\
\hline hsa05146 & Amoebiasis - Homo sapiens (human) & 0.001670065 & SERPINB3/SERPINB4/TGFB1 \\
hsa00830 & Retinol metabolism - Homo sapiens (human) & 0.01058397 & CYP3A5/DHRS3 \\
hsa05211 & Renal cell carcinoma - Homo sapiens (human) & 0.01089918 & ARNT2/TGFB1 \\
hsa05166 & HTLV-I infection - Homo sapiens (human) & 0.0230317 & EGR1/PPPR1/TGFB1 \\
hsa04933 & AGE-RAGE signaling pathway in diabetic complications & 0.02441325 & EGR1/TGFB1 \\
& Homo sapiens (human) & & \\
hsa04380 & Osteoclast differentiation - Homo sapiens (human) & 0.03999841 & PPP3R1/TGFB1 \\
hsa04068 & FoxO signaling pathway - Homo sapiens (human) & 0.04110769 & ATG12/TGFB1 \\
hsa04650 & Natural killer cell mediated cytotoxicity - & 0.04166677 & PPP3R1/SH3BP2 \\
& Homo sapiens (human) & & \\
hsa05322 & Systemic Lupus erythematosus - Homo sapiens (human) & 0.04166677 & HIST1H2AD/HIST2H2AA4 \\
\hline
\end{tabular}

REC8 inhibits EMT in gastric cancer cells through downregulation of EGR1 expression. When the REC8 overexpression plasmid was transfected into BGC823 cells, we detected an associated increase of E-cadherin, a decrease of SOX10 and vimentin at both the mRNA and protein levels (Fig. 3A and B; $\mathrm{P}=0.0049, \mathrm{P}=0.0031$ and $\mathrm{P}=0.0025$, respectively). We also observed a nuclear accumulation of $\beta$-catenin using immunofluorescence assays (Fig. 3C). Collectively, these results 
A

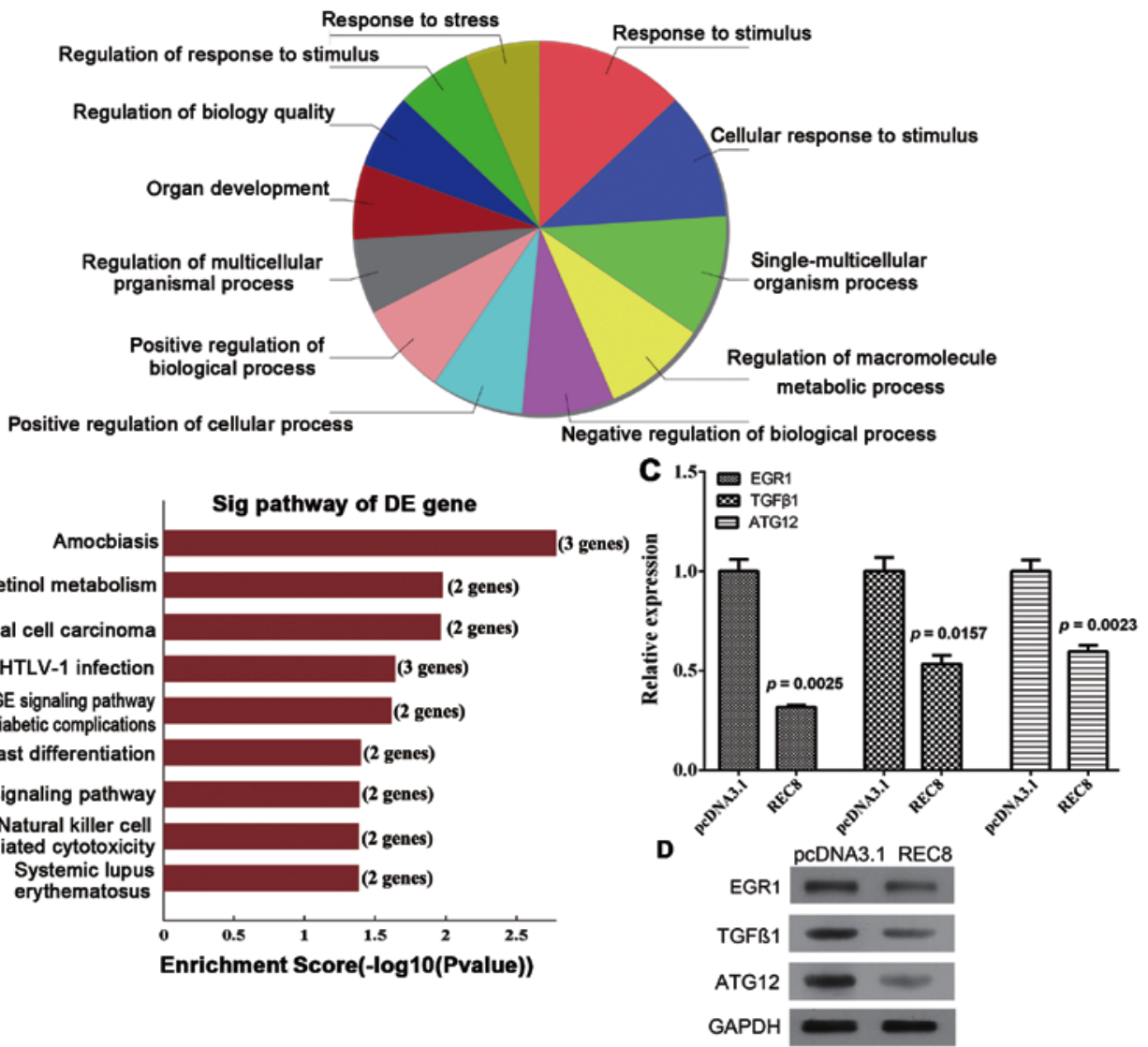

Figure 2. The expression of EGR1, TGF 11 and ATG12 is downregulated by REC8 overexpression. Gastric cancer cells BGC823 were transiently transfected with REC8 overexpression plasmid. (A) The pie chart revealed the top ten significant enrichment terms. The upper chart displayed the upregulated terms, while the lower one revealed the downregulated terms. (B) The signal pathway of DE gene was shown. (C) The mRNA expression levels of EGR1, TGF $\beta 1$ and ATG12 were assessed using qRT-PCR. (D) The protein levels of EGR1, TGF $\beta 1$ and ATG12 were assessed using western blotting.
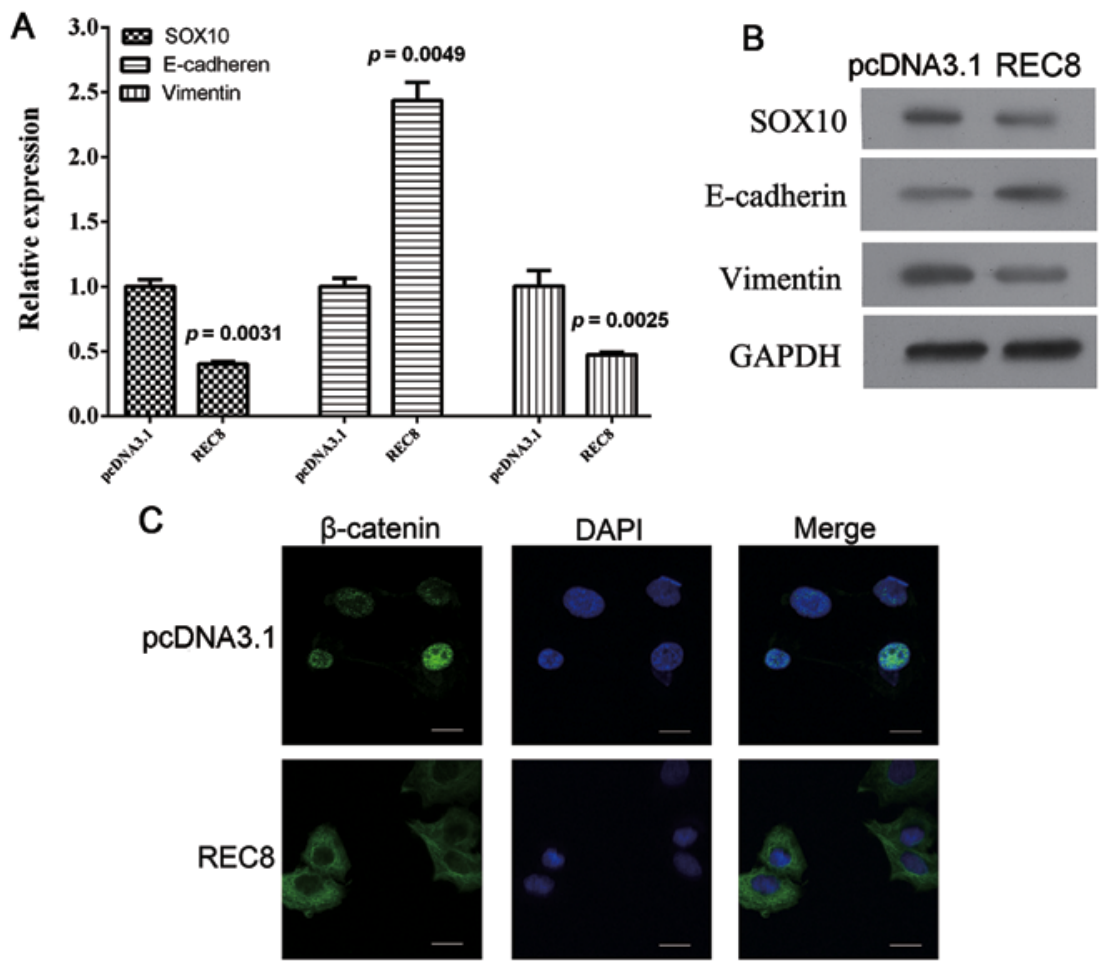

Figure 3. EMT is inhibited by REC8 overexpression. Gastric cancer cells BGC823 were transiently transfected with REC8 overexpression plasmid. (A) The mRNA expression levels of EMT markers (SOX10, E-cadherin and vimentin) were assessed using qRT-PCR. (B) The protein levels of EMT markers (SOX10, E-cadherin and vimentin) were assessed using western blotting. (C) The location of $\beta$-catenin in gastric cancer cells was assessed using immunofluorescence assay (magnification, $\mathrm{x} 400$ ). 

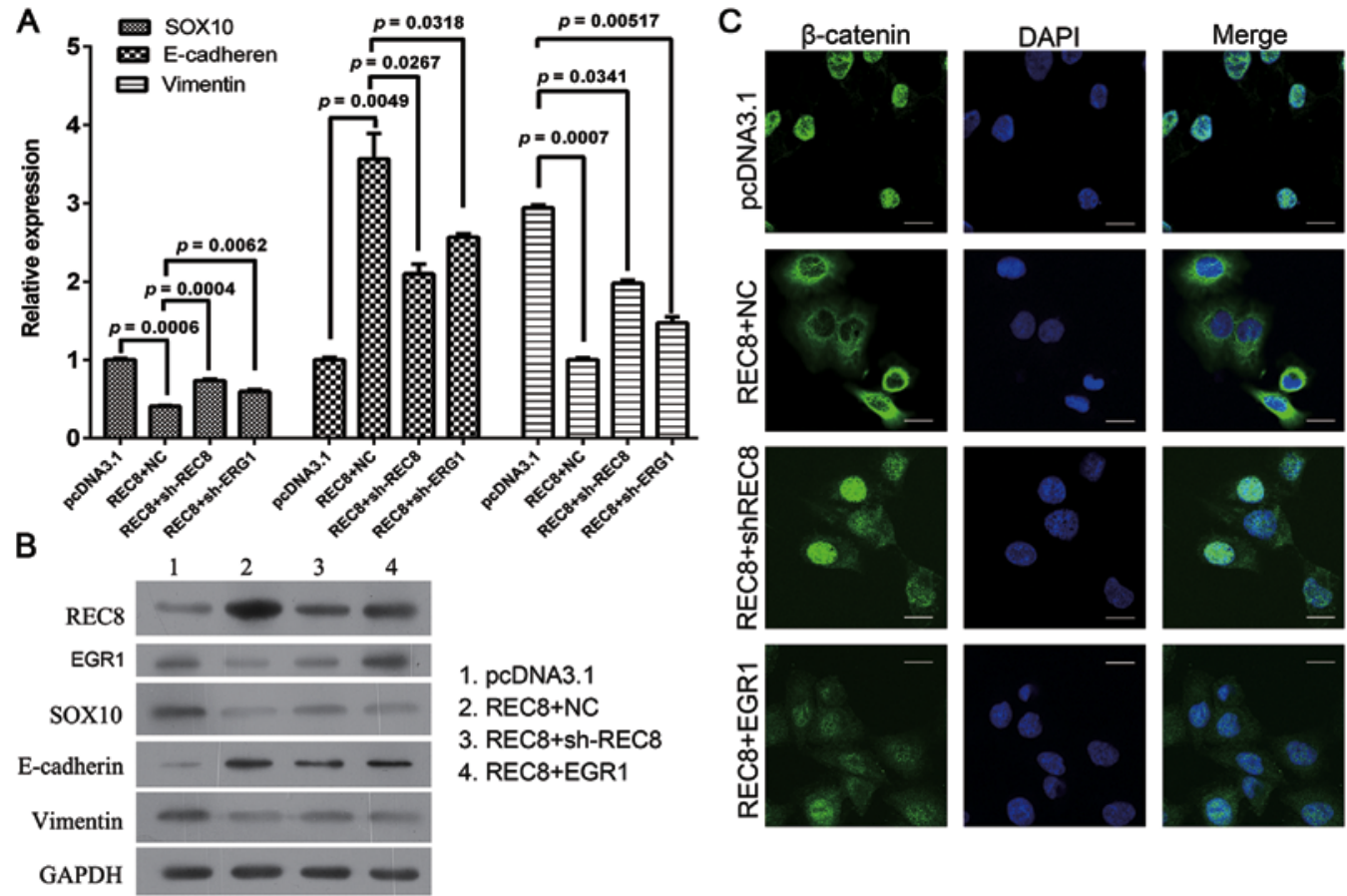

Figure 4. EMT inhibition induced by REC8 overexpression is reversed by REC8 ablation or EGR1 overexpression. REC8-overexpressed BGC823 cells were transiently transfected with REC8 shRNA or EGR1 overexpression plasmid. (A) The mRNA expression levels of EMT markers (SOX10, E-cadherin and vimentin) were assessed using qRT-PCR. (B) The protein levels of REC8, EGR1 and EMT markers (SOX10, E-cadherin and vimentin) were assessed using western blotting. (C) The location of $\beta$-catenin in gastric cancer cells was assessed using immunofluorescence assay (magnification, $\mathrm{x} 400$ ). All the results were expressed as the mean \pm SD of three independent experiments with triplicate wells.

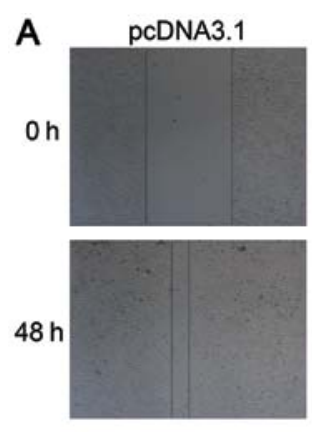

B

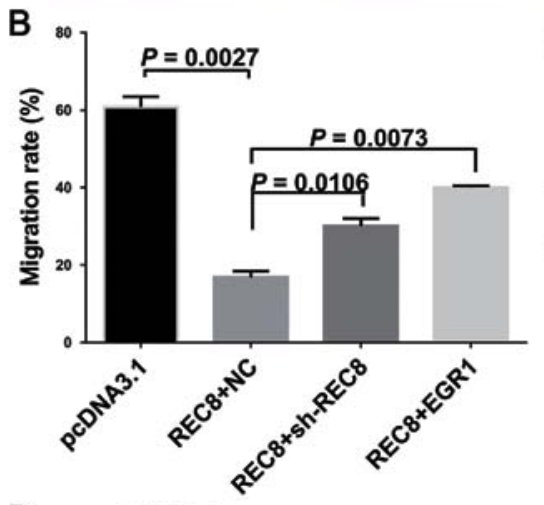

D

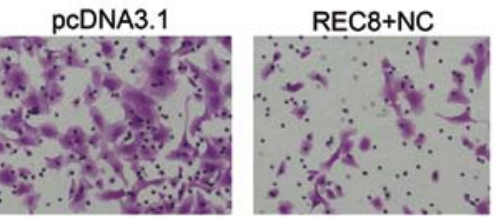

REC8+NC

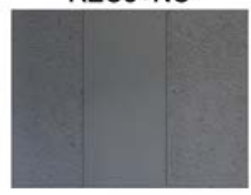

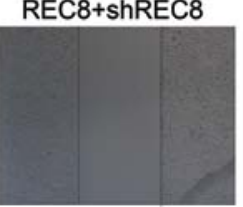
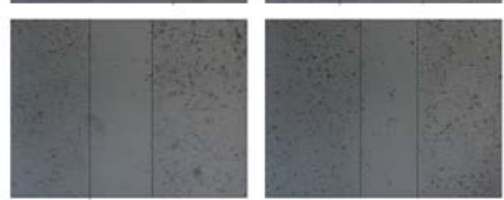

C
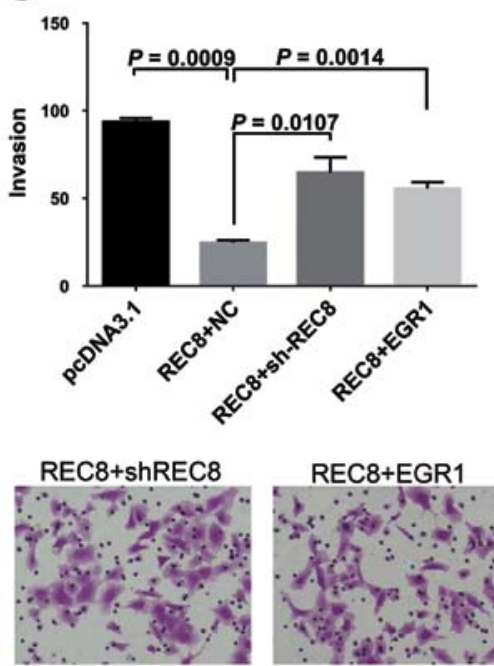

REC8+EGR1
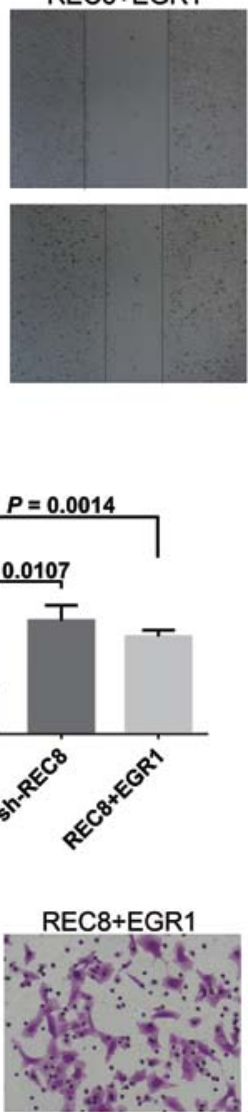

Figure 5. Mobility inhibition induced by REC8 overexpression is reversed by REC8 ablation or EGR1 overexpression. REC8-overexpressed BGC823 cells were transiently transfected with REC8 shRNA or EGR1 overexpression plasmid. (A and B) Cell migration was determined using wound healing migration assay (magnification, $\mathrm{x} 40$ ) and the width of wound was assessed using a microscope. (C and D) Cell invasion was determined using Transwell invasion assay and the number of cells were counted. All the results were expressed as the mean \pm SD of three independent experiments with triplicate wells. 


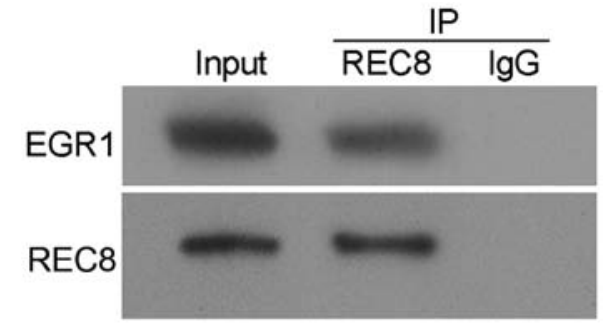

Figure 6. REC8 interacts with EGR1. The detection of the interaction between REC8 and EGR1 was determined by performing immunoprecipitation, and then EGR1 expression was detected using western blotting.

demonstrated that REC8 appeared to inhibit EMT in gastric cancer cells. In our previous microarray analysis, we found that REC8 regulated EGR1 expression. We, therefore transfected REC8-overexpressed BGC823 cells with REC8 shRNA or EGR1 overexpression plasmids and assessed the expression levels of REC8 and EGR1, and the expression characteristics of EMT markers using qRT-PCR, western blot and immunofluorescence assays. Consistent with the previous results obtained, the artificial overexpression of REC 8 decreased the expression of EGR1. When we ablated REC8 in REC8-transfected cells using REC8 shRNA or overexpressed EGR1 by using the pcDNA3.1-EGR1 plasmid, we found that the expression of REC8 or overexpressed EGR1 reduced the anti-EMT effect observed in REC8 overexpressed gastric cancer cells (Fig. 4). In addition, we examined whether REC8 regulated cell motility and invasion through EGR1 expression using the wound healing and the chamber invasion assays. When we ablated REC8 expression or overexpressed EGR1, we found that the anti-motility effect of REC8 in REC8 overexpressed gastric cancer cells was reduced, like we predicted (Fig. 5). These results revealed that REC 8 contributed to the anti-EMT effect through EGR1 expression.

REC8 and EGR1 physically interact and therefore REC8 regulates EGR1 expression. To further investigate the manner in which REC8 decreased EGR1 expression in gastric cancer cells, we performed an immunoprecipitation assay, and observed a strong interaction between REC8 and EGR1 (Fig. 6). This result revealed that REC8 decreased EGR1 expression by interacting with it.

\section{Discussion}

REC8 is an important component of the meiotic prophase chromosome axis that mediates sister chromatid cohesion, homologous chromosome pairing, crossover recombination and chromosome synapsis $(13,14)$. In the present study, we hypothesized that REC8 acts as a tumor-suppressor gene and we demonstrated for the first time that overexpressed REC8 inhibited gastric cancer cell proliferation and migration.

However, the regulatory mechanisms of REC8 in gastric cancer are not known. To understand how the carcinogenic process is influenced by REC 8 in gastric cancer, we searched for differential expression profiles and signaling pathways induced by REC 8 overexpression using microarrays. We ascertained that EGR1, TGF $\beta 1$ and ATG12 were downregulated by REC8 overexpression and were involved in the AGE-RAGE and FoxO signaling pathways. The FoxO pathway plays an important role in cell growth and cell survival (15), while the AGE-RAGE pathway is involved in EMT (16-18).

Gastric cancer is the second leading cause of cancerrelated deaths in the world (19). Even though patients undergo curative therapy, they still succumb to recurrent disease due to metastasis. Therefore, to treat gastric cancer, it is important to understand the molecular mechanisms underlying metastasis, thus finding the relevant therapeutic targets. EMT is a process where epithelial cells lose their characteristics including their spindle-cell shape, polarity, intercellular separation and pseudopodia formation (20-22). EMT is critical in many cancer biological behaviors, such as migration and invasion. The multifaceted REC8-mediated anticancer effects play a causal but unclear role in mammalian oncogenesis, thus in the present study, we focused on EMT and the AGE-RAGE pathway. Furthermore, EGR1, a protein in the AGE-RAGE pathway is an important factor in promoting EMT $(11,23)$. To explore the manner in which EGR1 expression promotes EMT in gastric cancer, we searched for EGR1 binding proteins using a protein interaction prediction program. We found that EGR1 interacted with SOX10, a marker of EMT. In support of our hypothesis, we observed marked changes in morphology, biochemistry, histochemistry and motility in BGC823-REC8 cells. Specifically, we observed an inhibition of EMT and a less motile and invasive phenotype than observed in vector cells. In previous studies, REC8 was considered to be a tumor suppressor via other factors and signaling pathways involved in the regulation of cell proliferation, apoptosis, the cell cycle and migration (1,24-33). Furthermore, based on data from epigenetic inactivation experiments, the antitumor effect of REC8 was attributed to methylation (34). As we previously described, EGR1 was demonstrated to be downregulated by REC8. Overexpression of EGR1 in REC8-transfected cells induced motility and EMT in gastric cancer cells which was the reverse of what was observed in REC8 overexpressed cells. These results revealed that EGR1 is a novel downstream protein of REC8 that regulates EMT in gastric cancer cells.

To understand the manner in which EGR1 expression was downregulated by REC8 in gastric cancer, we demonstrated that REC8 interacted with EGR1 and therefore downregulated EGR1. These results revealed that EGR1 promotes EMT depending on the amount of uncombined EGR1. The uncombined EGR1 can either bind with SOX10 directly or act as a transcription factor, and activate oncogenes to regulate EMT. The mechanism of REC8 in EMT in gastric cancer cells requires further study. In the present study we revealed that the EGR1 protein contributed to EMT regulation. In future research, the underlying mechanism of EGR 1 in the regulation of EMT needs to be illuminated more clearly.

In conclusion, our results revealed that REC8 decreased EMT in gastric cancer cells by regulating the expression of EGR1 and interacting with it. REC8 or EGR1 are potential targets in the treatment of gastric cancer.

\section{Acknowledgements}

The present study was supported by the National Natural Science Foundation of China (no. 81301758), the Natural Science Foundation of Guangdong (no. 2016A030310254) 
and the Postdoctoral Science Foundation of China (no. 2016M600648).

\section{Competing interests}

The authors declare that they have no competing interests.

\section{References}

1. Arseneault R, Chien A, Newington JT, Rappon T, Harris R and Cumming RC: Attenuation of LDHA expression in cancer cells leads to redox-dependent alterations in cytoskeletal structure and cell migration. Cancer Lett 338: 255-266, 2013.

2. Huang D, Duan H, Huang H, Tong X, Han Y, Ru G, Qu L, Shou C and Zhao Z: Cisplatin resistance in gastric cancer cells is associated with HER2 upregulation-induced epithelial-mesenchymal transition. Sci Rep 6: 20502, 2016.

3. Huang J, Xiao D, Li G, Ma J, Chen P, Yuan W, Hou F, Ge J, Zhong M, Tang Y, et al: EphA2 promotes epithelial-mesenchymal transition through the Wnt/ $\beta$-catenin pathway in gastric cancer cells. Oncogene 33: 2737-2747, 2014.

4. Dravis C, Spike BT, Harrell JC, Johns C, Trejo CL, SouthardSmith EM, Perou CM and Wahl GM: Sox 10 regulates stem/ progenitor and mesenchymal cell states in mammary epithelial cells. Cell Reports 12: 2035-2048, 2015.

5. Nasmyth K: Disseminating the genome: Joining, resolving, and separating sister chromatids during mitosis and meiosis. Annu Rev Genet 35: 673-745, 2001.

6. Laird PW: The power and the promise of DNA methylation markers. Nat Rev Cancer 3: 253-266, 2003.

7. Furuta J, Nobeyama Y, Umebayashi Y, Otsuka F, Kikuchi K and Ushijima T: Silencing of Peroxiredoxin 2 and aberrant methylation of $33 \mathrm{CpG}$ islands in putative promoter regions in human malignant melanomas. Cancer Res 66: 6080-6086, 2006.

8. Okamoto Y, Sawaki A, Ito S, Nishida T, Takahashi T, Toyota M, Suzuki H, Shinomura Y, Takeuchi I, Shinjo K, et al: Aberrant DNA methylation associated with aggressiveness of gastrointestinal stromal tumour. Gut 61: 392-401, 2012.

9. Liu D, Shen X, Zhu G and Xing M: REC 8 is a novel tumor suppressor gene epigenetically robustly targeted by the PI3K pathway in thyroid cancer. Oncotarget 6: 39211-39224, 2015.

10. Xu Y, Toure F, Qu W, Lin L, Song F, Shen X, Rosario R, Garcia J, Schmidt AM and Yan SF: Advanced glycation end product (AGE)-receptor for AGE (RAGE) signaling and up-regulation of Egr-1 in hypoxic macrophages. J Biol Chem 285: 23233-23240, 2010.

11. Sun S, Ning X, Zhai Y, Du R, Lu Y, He L, Li R, Wu W, Sun W and Wang H: Egr-1 mediates chronic hypoxia-induced renal interstitial fibrosis via the PKC/ERK pathway. Am J Nephrol 39: 436-448, 2014

12. Zhang H, Chen X, Wang J, Guang W, Han W, Zhang H, Tan X and Gu Y: EGR1 decreases the malignancy of human non-small cell lung carcinoma by regulating KRT18 expression. Sci Rep 4: $5416,2014$.

13. Yoon SW, Lee MS, Xaver M, Zhang L, Hong SG, Kong YJ, Cho HR, Kleckner N and Kim KP: Meiotic prophase roles of Rec 8 in crossover recombination and chromosome structure. Nucleic Acids Res 44: 9296-9314, 2016.

14. Watanabe $Y$ and Nurse $P$ : Cohesin Rec8 is required for reductional chromosome segregation at meiosis. Nature 400: 461-464, 1999.

15. Cohen S, Lee D, Zhai B, Gygi SP and Goldberg AL: Trim32 reduces $\mathrm{PI} 3 \mathrm{~K}-\mathrm{Akt}-\mathrm{FoxO}$ signaling in muscle atrophy by promoting plakoglobin-PI3K dissociation. J Cell Biol 204: 747-758, 2014.

16. Raghavan CT and Nagaraj RH: AGE-RAGE interaction in the TGF 32 -mediated epithelial to mesenchymal transition of human lens epithelial cells. Glycoconj J 33: 631-643, 2016.

17. Song JS, Kang CM, Park CK, Yoon HK, Lee SY, Ahn JH and Moon HS: Inhibitory effect of receptor for advanced glycation end products (RAGE) on the TGF- $\beta$-induced alveolar epithelial to mesenchymal transition. Exp Mol Med 43: 517-524, 2011.
18. Li JH, Wang W, Huang XR, Oldfield M, Schmidt AM, Cooper ME and Lan HY: Advanced glycation end products induce tubular epithelial-myofibroblast transition through the RAGE-ERK1/2 MAP kinase signaling pathway. Am J Pathol 164: 1389-1397, 2004.

19. Jemal A, Siegel R, Xu J and Ward E: Cancer statistics, 2010. CA Cancer J Clin 60: 277-300, 2010.

20. Hay ED: An overview of epithelio-mesenchymal transformation. Acta Anat (Basel) 154: 8-20, 1995.

21. Kalluri R and Weinberg RA: The basics of epithelial-mesenchymal transition. J Clin Invest 119: 1420-1428, 2009.

22. Thiery JP, Acloque H, Huang RY and Nieto MA: Epithelialmesenchymal transitions in development and disease. Cell 139: 871-890, 2009.

23. Ozen E, Gozukizil A, Erdal E, Uren A, Bottaro DP and Atabey N: Heparin inhibits hepatocyte growth factor induced motility and invasion of hepatocellular carcinoma cells through early growth response protein 1. PLoS One 7: e42717, 2012.

24. Zhang Z, Zhang B, Li W, Fu L, Fu L, Zhu Z and Dong JT: Epigenetic silencing of miR-203 upregulates SNAI2 and contributes to the invasiveness of malignant breast cancer cells. Genes cancer 2: 782-791, 2011.

25. Ying J, Srivastava G, Hsieh WS, Gao Z, Murray P, Liao SK, Ambinder R and Tao Q: The stress-responsive gene GADD45G is a functional tumor suppressor, with its response to environmental stresses frequently disrupted epigenetically in multiple tumors. Clin Cancer Res 11: 6442-6449, 2005.

26. Regel I, Eichenmüller M, Joppien S, Liebl J, Häberle B, Müller-Höcker J, Vollmar A, von Schweinitz D and Kappler R: IGFBP3 impedes aggressive growth of pediatric liver cancer and is epigenetically silenced in vascular invasive and metastatic tumors. Mol Cancer 11: 9, 2012.

27. Pruitt SC, Bailey KJ and Freeland A: Reduced Mcm 2 expression results in severe stem/progenitor cell deficiency and cancer. Stem Cells 25: 3121-3132, 2007.

28. Medina-Ramirez CM, Goswami S, Smirnova T, Bamira D, Benson B, Ferrick N, Segall J, Pollard JW and Kitsis RN: Apoptosis inhibitor ARC promotes breast tumorigenesis, metastasis, and chemoresistance. Cancer Res 71: 7705-7715, 2011.

29. Liu JY, Qian D, He LR, Li YH, Liao YJ, Mai SJ, Tian XP, Liu YH, Zhang JX, Kung HF, et al: PinX1 suppresses bladder urothelial carcinoma cell proliferation via the inhibition of telomerase activity and p16/cyclin D1 pathway. Mol Cancer 12: 148, 2013.

30. Kim MO, Lee YJ, Park JH, Ryu JM, Yun SP and Han HJ: PKA and cAMP stimulate proliferation of mouse embryonic stem cells by elevating GLUT1 expression mediated by the NF- $\kappa B$ and CREB/CBP signaling pathways. Biochim Biophys Acta 1820: 1636-1646, 2012.

31. Kabbout M, Garcia MM, Fujimoto J, Liu DD, Woods D, Chow CW, Mendoza G, Momin AA, James BP, Solis L, et al: ETS2 mediated tumor suppressive function and MET oncogene inhibition in human non-small cell lung cancer. Clin Cancer Res19: 3383-3395, 2013

32. Du W, Jiang P, Mancuso A, Stonestrom A, Brewer MD, Minn AJ, Mak TW, Wu M and Yang X: TAp73 enhances the pentose phosphate pathway and supports cell proliferation. Nat Cell Biol 15: 991-1000, 2013.

33. Deep G, Jain AK, Ramteke A, Ting H, Vijendra KC, Gangar SC, Agarwal C and Agarwal R: SNAI1 is critical for the aggressiveness of prostate cancer cells with low E-cadherin. Mol Cancer 13: 37, 2014.

34. Yu J, Liang Q, Wang J, Wang K, Gao J, Zhang J, Zeng Y, Chiu PW, $\mathrm{Ng}$ EK and Sung JJ: REC8 functions as a tumor suppressor and is epigenetically downregulated in gastric cancer, especially in EBV-positive subtype. Oncogene 36: 182-193, 2017.

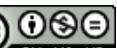

This work is licensed under a Creative Commons Attribution-NonCommercial-NoDerivatives 4.0 International (CC BY-NC-ND 4.0) License. 